\title{
Deliberate use of placebos in clinical practice: what we really know
}

Cory S Harris, ${ }^{1,2}$ Amir Raz ${ }^{2,3}$

Increasingly a focus of research as well as media reports and online forums, the use of placebos in clinical medicine extends beyond sugar pills and saline injections. Physician surveys conducted in various countries invariably report that placebos are routinely used clinically, impure placebos more frequently than the pure ones, and that physicians consider them to be of legitimate therapeutic value. Inconsistent study methodologies and physician conceptualisations of placebos may complicate the interpretation of survey data, but hardly negate the valuable insights these research findings provide. Because impure placebos are often not recognised as such by practitioners, they remain at the fringe of many placebo-related debates, hence quietly absent from discussions concerning policy and regulation. The apparent popularity of impure placebos used in clinical practice thus presents unresolved ethical concerns and should direct future discussion and research.

Converging independently replicated evidence suggests that physicians do use placebos in clinical practice. Regardless of definitions and conceptualisations, these accounts suggest that, unlike most people, many clinicians construe placebos as more than inert 'nothings'. The tendency to equate placebos with nothing is rather simplistic because it sweepingly dismisses large bodies of research concerning active placebos, impure placebos and what placebos should or should not contain. The field of placebo science needs to unpack a vast entanglement of parameters, including psychosocial factors, such as expectations, suggestions, symbolic thinking, framing and cultural influences. This task requires an appreciation of both the social and life sciences, and a nuanced approach to the use of placebos in the clinic.

Findings from recent surveys of healthcare professionals from several countries propose that placebos are being used clinically; however, conceptual and methodological variations complicate the interpretation of results and comparisons between studies. ${ }^{1}$ While media headlines

\footnotetext{
${ }^{1}$ School of Dietetics and Human Nutrition, McGill University, Montreal, Canada; ${ }^{2}$ Lady Davis Institute for Medical Research of the Jewish General Hospital, Montreal, Canada; ${ }^{3}$ Departments of Psychiatry, Neurology \& Neurosurgery, and Psychology McGill University, Montreal, Canada

Correspondence to Dr Amir Raz, Duff Medical Building, \#103 at the Montreal Neurological Institute, 3775 University Street, Montreal, Quebec H3A 2B4, Canada; amir.raz@mcgill.ca
}

rarely tell the whole story, we should not be too quick to dismiss what these studies tell us about the use of placebos in clinical care today. Conceptual differences on placebo abound in the literature, notably, inconsistent survey definitions and physician understandings of the terms 'placebo', 'placebo response', and 'placebo effect'. This potential confusion, however, is more than an artefact; it reflects an ongoing debate regarding the role of placebos in clinical practice and research. Whereas to some professionals the definition of 'placebos as inert substances' feels most appropriate, broader conceptualisations are widespread. For example, in surveys that provided physicians with a choice of definition for placebo, 'an intervention that is inert or innocuous' was by far the least popular answer. ${ }^{2}{ }^{3}$ This trend may well stem from, but certainly goes far beyond, the tacit understanding of, and acquiescence to, placebos as zero. Physician perceptions notwithstanding, prescribing either inert or active interventions that are patently unsubstantiated poses a serious ethical conundrum.

According to systematic reviews, physicians report using impure placebos more frequently than pure placebos, with only a small percentage turning to sugar pills or saline injections. ${ }^{14}$ For example, only $20 \%$ of physicians surveyed in Canada reported using placebos, while fewer prescribed inert substances, and many more used at least one form of treatment 'in situations without demonstrated or expected clinical efficacy'. ${ }^{2}$ Interestingly, US ${ }^{3}$ and Danish ${ }^{5}$ studies, respectively, report that $45 \%$ and $86 \%$ of physicians use placebos, but when asked directly about treatment type, physicians in both countries attest to using pure and impure placebos in proportions comparable with those described in the Canadian study. Regardless of headlines, which typically portray placebos as inactive pills, overarching trends indicate that clinicians more often dispense impure, rather than pure, placebos.

\section{PURITY MATTERS}

The observation that physicians opt for impure placebos rather than pure placebos is disconcerting because most ethical debates and professional policies focus squarely on pure placebos. Clearly, deliberately prescribing inert tablets differs in many ways from prescribing active treatments, which may benefit the patient despite a lack of supporting evidence. However, because physicians accept the benefit of placebo-like interventions, ${ }^{2}{ }^{67}$ these two examples share much in common. Moreover, unless fully disclosed to the patient, prescriptions for unproven active interventions infringe on patient autonomy and informed consent in the same way that pure placebos do, yet the active nature of these medicines also carries the risk of adverse effects.

Whereas physician surveys identify global patterns in the clinical use of placebos, interpretation of regional differences-conceptually and practicallymust consider relevant sociocultural and political environments. Despite the dominance of modern biomedicine in all recently surveyed countries, alternative medical systems integrate alongside, or otherwise coexist with, conventional medicine to varying degrees. National healthcare models also differ, for example, publicly versus privately funded systems, as do legal and regulatory frameworks, professional codes and the social values in which they evolved. Placebo effects vary as a function of country and culture. ${ }^{8}$ Perhaps such cultural variations lend colour to physician perceptions and the use of different types of placebos-for example, contributing to the tendency of American physicians to prescribe antibiotics as placebos, while their colleagues in Canada and Denmark tend to prescribe vitamins. ${ }^{2} 35$

Qualitative methods, to complement our quantitative approaches, are necessary to better capture the nuanced views of practicing physicians. And yet, we continue to turn to quantitative studies in order to identify new and pressing directions for future research. For example, 
Canadian psychiatrists are six times more likely than non-psychiatrist physicians to prescribe sub-therapeutic doses of drugs to patients. In light of the incremental dosing practices associated with antidepressants, and the continuing controversy over their clinical efficacy for mild-to-moderate depression, our team interviewed psychiatrists to explore notions of sub-therapeutic doses and to shed light on their role-as well as that of placebo effects-in the clinical treatment of depression. Briefly, none of the interviewed psychiatrists equated sub-therapeutic doses with placebos, but several acknowledged that placebo effects likely contribute to positive patient responses following ingestion of very low doses. Furthermore, physicians appear to promote placebo effects through their interactions with patients, consciously crafting language around the topic of dose to shape expectancies of both improvement and adverse effects. ${ }^{9}$ Whereas psychiatrists may shy away from explicitly invoking placebo effects, elements of placebo medicine retain an essential place in modern psychiatry.

\section{CONCLUSION}

Physician surveys from Europe, North America and the Middle East consistently report the clinical use of placebospredominantly impure and active placebos. Regardless of methodological discrepancies, these studies underscore the need to expand ethical debates on the clinical use of placebos beyond inert treatments. With conceptual inconsistencies leaving many physicians unaware of the fact that they prescribe impure placebos, ${ }^{2} 910$ educating medical students about the science of placebos-a topic rarely taught-is a fundamental step towards clarifying misconceptions, aligning terminologies and making ethical and judicious use of placebos in clinical practice. ${ }^{11}$ From the American Medical Association's restrictive position to the German Medical Association's permissive stance, policies regulating the use of placebos are nascent and controversial when they are not entirely absent, as is the case in Canada. This May, stakeholders ranging from physicians and scholars to policy makers and lawyers will convene in Montreal to seek consensus and to forward Canadian policy recommendations. Only through such opportunities to share knowledge and exchange perspectives can we tackle the complex nature and farreaching implications of the use of placebos in the clinic.

- Do media headlines oversimplify the nature and prevalence of the use of placebos in modern medicine? Without question.

- Do existing survey data, with their inclusion of both pure and impure placebos, inflate the perceived use of inert treatments? Most likely.

- Do methodological differences between studies obscure direct comparison of results? To some extent.

- Should we disregard the insights gained from this research for these reasons? Absolutely not.

Contributors CSH is the lead author. AR contributed significantly to developing the arguments and writing the text.

Funding CSH is supported by a Canadian Institutes of Health Research Banting Postdoctoral Fellowship.

Competing interests None.

Provenance and peer review Commissioned; internally peer reviewed.

Received 30 March 2012

Accepted 31 March 2012
Published Online First 28 May 2012

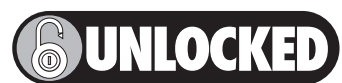

This paper is freely available online under the BMJ Journals unlocked scheme, see http://jme.bmj.com/site/ about/unlocked.xhtml.

J Med Ethics 2012;38:406-407.

doi:10.1136/medethics-2012-100695

\section{REFERENCES}

1. Louhiala P. What do we really know about the deliberate use of placebos in clinical practice? J Med Ethics 2012;38:403-50.

2. Raz A, Campbell N, Guindi D, et al. Placebos in clinical practice: comparing attitudes, beliefs, and patterns of use between academic psychiatrists and nonpsychiatrists. Can J Psychiatry 2011;56:198-208.

3. Sherman R, Hickner J. Academic physicians use placebos in clinical practice and believe in the mindebody connection. J Gen Intern Med 2008;23:7-10.

4. Fassler M, Meissner K, Schneider A, et al. Frequency and circumstances of placebo use in clinical practice-a systematic review of empirical studies. BMC Med 2010;8:15.

5. Hrobjartsson A, Norup M. The use of placebo interventions in medical practice-a national questionnaire survey of Danish clinicians. Eval Health Prof 2003;26:153-65.

6. Fassler M, Gnadinger M, Rosemann T, et al. Placebo interventions in practice: a questionnaire survey on the attitudes of patients and physicians. $B r \mathrm{~J}$ Gen Pract 2011;61:101-7.

7. Sherman R, Hickner J. Academic physicians use placebos in clinical practice and believe in the mindbody connection. J Gen Intern Med 2008;23:7-10.

8. Moerman DE. Cultural variations in the placebo effect: ulcers, anxiety, and blood pressure. Med Anthropol 0 2001;14:51-72.

9. de Jong $\mathbf{V}$, Raz A. Sub-Therapeutic doses in the treatment of depression: the implications of Starting low and going Slow. J Mind-Body Regul 2011;1:73-84.

10. Fent R, Rosemann $T$, Fassler $M$, et al. The use of pure and impure placebo interventions in primary care-a qualitative approach. BMC Fam Pract 2011;12:11.

11. Raz A, Guindi D. Placebos and medical education McGill J Med 2008;11:223-6. 